\title{
Ingestion of Goji Berry (LyciumBarbarum) Evaluation on Plasma Levels of Total Cholesterol, Lipid Fractions, Glycaemia, Serotonin and Arterial Pressure
}

\author{
Sonia R Jurado*, Beatriz C Leão, Silvana B de Oliveira, Daiani Rosa, Francieli S da Costa, Tatiane F dos \\ Santos
}

Department of Public Health, Federal University of Mato Grosso do Sul, TrêsLagoas, Avenue Ranulpho Marquês Leal, Brazil.

"Corresponding author: Sonia R Jurado, Department of Public Health, Federal University of Mato Grosso do Sul, TrêsLagoas, Avenue Ranulpho Marquês Leal, Brazil. Tel: +5506735093714; E-Mail: srjurado@bol.com.br

Citation: Jurado SR, Leão BC, de Oliveira SB, Rosa D, da Costa FS, et al. (2017)Ingestion of Goji Berry (LyciumBarbarum) Evaluation on Plasma Levels of Total Cholesterol, Lipid Fractions, Glycaemia, Serotonin and Arterial Pressure. Cardiolog Res Cardiovasc Med 2: 116. DOI: 10.29011/2575-7083.000016

Received Date: 10 May, 2017; Accepted Date: 31 May, 2017; Published Date: 8 June, 2017

\begin{abstract}
Goji Berry is the name of the fruits of the plant Lyciumbarbarum. Goji berry contains 19 amino acids, 21 minerals, broad spectrum of carotenoids, $\beta$-sitosterol (anti-inflammatory), vitamins A, C and E, among other substances. The objective of this study was to evaluate the levels of lipid fractions, total cholesterol, serotonin levels, blood pressure, body weight and abdominal circumference, before and after the ingestion of goji berry in women. Twenty women, aged between 18 and 50 years, participated in the study. They had the data evaluated: body weight, height, abdominal circumference and blood pressure. Participants received 40 grams of dehydrated goji berry, consumed in two daily servings of 20 grams, one at breakfast and the other after lunch, for 15 days. Laboratory tests were performed to evaluate total cholesterol, lipid fractions, glycaemia and serotonin, on day 0 and 16 of the study. Daily consumption of goji berry provided general well-being, decreased abdominal circumference, body weight LDL, glycaemia, systolic and diastolic blood pressure and increased serotonin, and may be indicated as a complementary treatment for hypertension, diabetes and depression.
\end{abstract}

Keywords: Blood Pressure; Cholesterol; Glycaemia;Goji Berry; Serotonin

\section{Introduction}

Functional foods are defined as any substance or component of a food that provides health benefits, including the prevention and treatment of diseases. Some parameters should be taken into account in relation to functional foods. For Borges [1], they must exert a metabolic or physiological effect that contributes to the physical health and the reduction of the risk of development of chronic diseases. Therefore, functional foods should be part of the daily diet to prevent and control diseases.

Fruit consumption can reduce the risk of cardiovascular diseases, mainly because they contain antioxidants. The best-known antioxidants are the polyphenols and vitamin $\mathrm{C}$ found in goji berry.
Goji berry is the name of the fruits of the plant Lyciumbarbarum. The plant originates from the mountains of Tibet. Goji berry contains 19 amino acids, 21 minerals, a wide range of carotenoids ( $\beta$-Carotene, Neoxanthin, Cryptoxanthin, Zeaxanthin), polysaccharides, flavonoids, phytosterols, $\beta$-sitosterol (anti-inflammatory), vitamins $\mathrm{A}, \mathrm{C}$ and $\mathrm{E}$ (tocopherol) among other substances [2,3].

Goji berry has several biological activities, including effects on aging [4], neuroprotection [5-7], anti-fatigue/resistance, increased metabolism, welfare promotion, immunomodulation [8], antitumor activity [9-11], glycemic control in diabetics [12] and treatment of osteoporosis with improved bone density and matrix [13]. Tocopherols have been shown to have positive effects on patients with HIV and those with Parkinson syndrome. Phytosterols have been found to reduce the low-density lipoprotein cholesterol level, and increase the high-density lipoprotein cholesterol level in the blood [14]. 


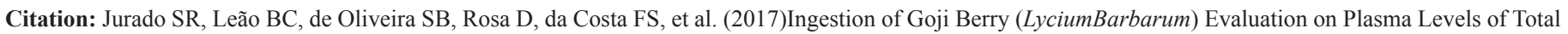
Cholesterol, Lipid Fractions, Glycaemia, Serotonin and Arterial Pressure. Cardiolog Res Cardiovasc Med 2: 116. DOI: 10.29011/2575-7083.000016

The s-1 polysaccharides (LBP) extracted from Lyciumbarbarumcan be used in the treatment of diabetes. In vitro and in vivoexperiments showed that LBP s-1 promoted significant hypoglycemic effects, with increased insulin secretion and pancreatic $\beta$-cell proliferation [15].

Goji berry has high antioxidant power and therefore helps in the prevention of diseases related to oxidative stress in humans. Goji effects were investigated in a 30-day, randomized, doubleblind, placebo-controlled study of a population of 50 healthy Chinese adults aged 55-72 years. Serum levels of the following antioxidants were evaluated: Superoxide Dismutase (SOD), Glutathione Peroxidase (GSH-Px) and lipid peroxidation (indicated by decreased levels of Malondialdehyde, MDA) before and after consumption of goji or placebo. In the goji group, antioxidants increased significantly from $8.4 \%$ for SOD and $9.9 \%$ for GSH-Px between pre-treatment and post-treatment, while MDA was significantly lower in $8.7 \%$ [16].

The consumption of goji berry has increased worldwide in recent years; however, more clinical studies are needed for its knowledge on human health. The objective of the present study was to evaluate the lipid profile, glycaemia, serotonin, arterial pressure and abdominal circumference before and after the ingestion of goji berry (Lyciumbarbarum) in women.

\section{Material and Methods}

Twenty female participants, aged 18-50, were selected. All the participants signed informed consent and had the following anthropometric data evaluated: body weight, height, abdominal circumference and blood pressure.

In this study, those who used anticoagulants, goji berry allergy, pregnant women or infants were excluded. Also excluded were women who were taking medication for dyslipidemia and had the following diseases Diabetes mellitus, nephrotic syndrome, acute or chronic renal failure, hypo or hyperthyroidism, hormone replacement therapy or other pathology capable of altering the lipid profile.

The guidelines of the Brazilian Society of Diabetes [17] and the Brazilian Society of Cardiology [18] were used for glycaemia and dyslipidemias, defined as normal glycaemia $<110 \mathrm{mg} / \mathrm{dl}$, hyperglycemia $\geq 126 \mathrm{mg} / \mathrm{dl}$ and individuals with decreased glucose tolerance $\geq 110 \mathrm{mg} / \mathrm{dl}$ and lower than $126 \mathrm{mg} / \mathrm{dl}$; for high triglycerides, values $>200 \mathrm{mg} / \mathrm{dl}$; altered cholesterol $>200 \mathrm{mg} / \mathrm{dl}$ and hypercholesterolemia $>240 \mathrm{mg} / \mathrm{dl}$.

Laboratory tests were performed to evaluate glycaemia, total cholesterol, triglycerides, HDL and LDL, on day 0 and 16 of the study. Participants received 40 grams of dehydrated goji berry, consumed in two daily servings of 20 grams, one at breakfast and the other after lunch, for 15 days. All participants answered, at the end of the study, a questionnaire containing questions about pos- sible beneficial and side effects of goji berry consumption.

Statistical analysis of the following measurements arterial pressure, lipid fractions, glycaemia, serotonin, abdominal circumference and body weight before and after goji consumption were performed. The $t$ test was used and values of $p$ less than 0.05 were considered statistically significant.

\section{Results}

There was a significant increase in serotonin levels and significant reduction in abdominal circumference, total cholesterol, LDL, blood glucose, and diastolic and systolic arterial pressure. Differences in triglyceride levels, HDL and body weight were detected before and after the goji consumption, however, these were not significant (Table 1).

\begin{tabular}{|c|c|c|c|}
\hline Parameter & Day 0 & Day 16 & $\mathrm{p}$ \\
\hline Weight body (Kg) & $79.0 \pm 3.5$ & $78.4 \pm 3.4$ & 0.007 \\
\hline $\begin{array}{c}\text { Abdominal circumfer- } \\
\text { ence (cm) }\end{array}$ & $95.1 \pm 2.6$ & $91.8 \pm 2.9$ & $<0.0001$ \\
\hline $\begin{array}{c}\text { Systolic arterial pressure } \\
\text { (mmHg) }\end{array}$ & $120.0 \pm 2.5$ & $111.5 \pm 2.7$ & 0.0017 \\
\hline $\begin{array}{c}\text { Diastolic arterial pres- } \\
\text { sure (mmHg) }\end{array}$ & $83.5 \pm 2.7$ & $75.0 \pm 3.0$ & 0.0037 \\
\hline Cholesterol (mg/dl) & $183.6 \pm 8.5$ & $174.8 \pm 9.1$ & 0.06 \\
\hline HDL (mg/dl) & $47.1 \pm 2,7$ & $49.3 \pm 2.1$ & 0.06 \\
\hline LDL (mg/dl) & $156.7 \pm 24.2$ & $109.2 \pm 17.0$ & 0.02 \\
\hline Triglyceride (mg/d) & $169.6 \pm 27.6$ & $172.9 \pm 23.1$ & 0.44 \\
\hline Glucose (mg/dl) & $89.9 \pm 3.4$ & $85.0 \pm 2.8$ & 0.0007 \\
\hline Serotonin levels (ng/ml) & $54.5 \pm 3.4$ & $93.1 \pm 8.2$ & 0.04 \\
\hline
\end{tabular}

Table 1: Anthropometric Data, Diastolic and Systolic Blood Pressure and Serotonin Levels from the 20 Participants, Before (Day 0) and After (Day 16) the Consumption of Goji Berry. Data Expressed as Mean \pm Standard Error.

Participants reported subjective feeling of the satiety, increased energy level, sleep quality, ease to wake up, improvement in concentration, calmness, well-being, contentment, happiness, reduced fatigue and stress and improved the regularity of gastrointestinal function.

\section{Discussion}

Amagase and Nance [8]. showed that Lyciumbarbarum increased metabolic rate and reduced body weight in adults, as well as contributed to general well-being. These authors detected a significant mean reduction of $5.5 \pm 0.8 \mathrm{~cm}$ in the abdominal circumference of the subjects who consumed goji berry juice for 14 days (valor de p). Another study that evaluated the consumption of $120 \mathrm{ml} /$ day of goji berry juice in overweight adults for 14 days also showed a significant reduction in abdominal circumference, with a mean loss of $5.54 \pm 0.65 \mathrm{~cm}$. It is noteworthy that the group that consumed goji 


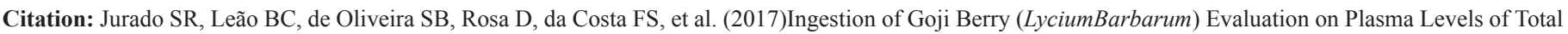
Cholesterol, Lipid Fractions, Glycaemia, Serotonin and Arterial Pressure. Cardiolog Res Cardiovasc Med 2: 116. DOI: $10.29011 / 2575-7083.000016$

berry was also subjected to a daily intake of 1,200 calories. The placebo group also underwent caloric restriction and had a loss of $0.88 \pm 0.83 \mathrm{~cm}$ of abdominal circumference throughout the experiment [19]. It was demonstrated in the present study a significant difference in abdominal circumference before and after consumption of L. barbarum for 15 days, with a reduction of $3.3 \mathrm{~cm}$.

Obesity is a worldwide public health problem and is associated with increased morbidity and mortality. Excess abdominal fat is strongly associated with increased cardiometabolic risk, cardiovascular problems and morbidity. One of the criteria for the diagnosis of the metabolic syndrome includes the measurement of abdominal circumference because the increased visceral adipose tissue is one of the triggers of the syndrome [20]. Therefore, daily consumption of goji berry may be indicated for reduction of abdominal circumference and decreased risk for metabolic and cardiac diseases.

Luo,et al. [21] reported that crude or purified polysaccharides (LPB) from L. barbarum can reduce blood glucose levels and serum concentrations of Total Cholesterol (TC) and Triglycerides (TG), while increasing High-Density Lipoprotein Density (HDL) in diabetic rabbits induced by alloxan. The results of another study also indicated that treatment with LBP for 28 days resulted in a significant decrease in the concentration of glucose, CT and TG levels in diabetic alloxan-induced rats [22]. These effects are due to LBP, the main bioactive constituents of L. barbarum.

Our study detected a significant reduction of glucose from $89.9 \pm 3.4$ to $85.0 \pm 2.8 \mathrm{mg} / \mathrm{dl}$ and $\mathrm{LDL}$ from $156.7 \pm 24.2$ to $109.2 \pm 17.0 \mathrm{mg} / \mathrm{dl}$ in women that consumed $40 \mathrm{~g} /$ day of $L$. barbarum for 15 days. There was a reduction of cholesterol from $183.6 \pm 8.5$ to $174.8 \pm 9.1 \mathrm{mg} / \mathrm{dl}$ in the participants, however, this difference was not statistically significant. An in vitro study revealed that the polysaccharide LBP-1 protects pancreatic cells producing insulin and also acts on insulin-resistant cells, facilitating the entry and consumption of glucose by the cells, contributing to the hypoglycemic effect of L. barbarum [23].

One study conducted in 67 patients with type 2 diabetes, 30 in the control group and 37 in the experimental group, who received $300 \mathrm{mg} /$ day of LPB for three months, showed a reduction in glycaemia, total cholesterol, triglycerides, LDL and increase in HDL, in the experimental group [24]. In this study, it was found that HDL increase in the participants after consumption of L. barbarum for 15 days, however, was not significant. HDL plays an important role in the transport of cholesterol from the peripheral blood to the liver via a reverse cholesterol transport pathway. Therefore, increased HDL is associated with a decreased risk of heart disease [25].

Adipokines are cytokines or hormones produced by adipocytes. It is reported that some adipokines such as TNF $\alpha$, leptin, IL-6 and ADPN are related to insulin resistance and subsequent development of type 2 diabetes [26]. Individuals with type 2 diabetes who used LPB had lower amounts of TNF $\alpha$, increased glucose uptake into cells and decreased insulin resistance [24].

One study evaluated the effects of L. barbarum LBP on fatigue after physical exercise. The model chosen was swimming in mice with weight overload and consumed LBP at the oral dose of $20 \mathrm{mg} /$ day and $60 \mathrm{mg} /$ day for a period of 14 days. It has been found that LBP can significantly prolong swimming time with weight overload, increase hepatic glycogen content, retard and prevent the increase of lactic acid in the blood as well as assist in the faster recovery of fatigue in these animals [27]. The best results were obtained with daily consumption of $60 \mathrm{mg} /$ day LBP, when compared to the $20 \mathrm{mg}$ /day LBP and control group of animals that did not receive LBP [27].

It is known that the body's resilience has declined sharply if the power is depleted. Because glycogen is the most important energy source during exercise, increasing the glycogen stored in the liver is an advantage for resistance to exercise. Increased glycogen consumption and accumulation of urea and other metabolites cause body fatigue [28]. One study demonstrated that LBP ingestion of L. barbarum improved the physical performance and energy metabolism of rats with weight overload during swimming [27].

In our study, the participants reported as subjective feeling the reduction of fatigue. However, more clinical studies are needed to better understand the anti-fatigue mechanism of $L$. barbarum LBP. Goji berry also has vascular effects. A study carried out with rat aorta rings that consumed LBP detected increased expression of Endothelial Nitric Oxide Synthase (eNOS) and increased nitric oxide, a vasodilator agent produced by the endothelium of blood vessels. Therefore, LBP helps reduce blood pressure by increasing the production of nitric oxide and vasodilation [29]. The consumption of goji berry for 15 days by participants in this study significantly reduced systolic and diastolic blood pressure.

It is known that administration of goji berry $(150 \mathrm{mg} / \mathrm{kg} /$ day and $300 \mathrm{mg} / \mathrm{kg} /$ day) improves testoterone levels and sexual activity in rats of advanced age. The hypothesis is that goji berry prevents the decrease of serum testosterone due to its antioxidant effect. In addition, it increases the expression of eNOS, Neural Nitric Oxide Synthase (nNOS) and Cyclic Guanosine Monophosphate (cGMP), subsequently inhibiting apoptosis in muscle cells and deposition of collagen in the penis, improving penile erection and sexual function in aged rats [30].

In addition, the effects of well-being, tranquility, and happiness attributed to goji are possibly due to tryptophan, the precursor of serotonin, in the Central Nervous System and maintenance of catecholamine levels [31]. L. barbarum is indicated as an auxiliary treatment for depression, a condition that affects $10 \%$ of the world population [32]. Daily diet with goji berry can also have positive effect in preventing this disease. In addition, this fruit also contains 


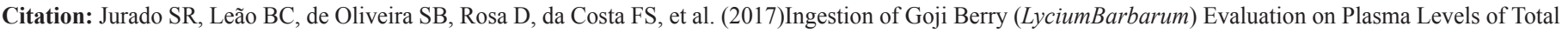
Cholesterol, Lipid Fractions, Glycaemia, Serotonin and Arterial Pressure. Cardiolog Res Cardiovasc Med 2: 116. DOI: 10.29011/2575-7083.000016

safranal, an aromatic compound, with anti-depressive properties and which acts as a GABAA receptor agonist [31].

The effects of Lycium barbarum were measured on the wellbeing of healthy adults (18-60 years of age), who consumed a standardized juice of goji berry. Subjects consumed $120 \mathrm{ml} /$ day of goji berry or placebo for 30 days. The researchers applied questions with a scale of 0-5 for general well-being, stress, depression and plasma levels of catecholamines (epinephrine and norepinephrine) before and after the intervention [33]. [Higher study characteristics].

Amagase and Nance [33] showed a significant improvement in sleep quality and ease of wakefulness, as well as a significant reduction in concentration difficulty, excessive worry, memory loss, and fatigue after exercise in the goji berry group $(n=20)$. The placebo group $(n=19)$ did not show significant changes, but there was a tendency to increase stress and depression. Plasma levels of epinephrine and norepinephrine in the placebo group, compared to pre-intervention, increased significantly by $20 \%$ and $31 \%$, respectively, whereas the goji berry group did not show significant changes. These results suggest that daily consumption of goji berry increases subjective feelings of general well-being and decreases stress and depression, which may be related to stabilization of catecholamine levels [33].

A meta-analysis study including 161 participants, allocated to two groups: experimental $(\mathrm{n}=81)$ and placebo $(\mathrm{n}=80)$, in which the experimental group consumed $120 \mathrm{ml}$ of goji berry juice per day, demonstrated welfare and neurological and psychological performance in the group that consumed goji berry when compared to placebo [34].

\section{Conclusions}

Our results indicate the following clinical results of goji berry, LDL reduction, decreased arterial pressure, blood glucose, abdominal circumference, and increased feelings of well-being. The benefits of goji berry are numerous for health and because of its nutritional components it is the best functional food known to date and can be used in the prevention and treatment of type 2 diabetes, hypertension, overweight and obesity, hypercholesterolemia and depression.

\section{References}

1. Borges VC (2001) Functional foods prebiotics probiotics phytochemicals and symbiotic. In: Waitzberg DL. Enteral and Parenteral Nutrition in Clinical Practice. Sao Paulo: Atheneu.

2. Ulbricht C,Bryan JK, Costa D, Culwell S, Giese N, et al. (2014)An evidence-based systematic review of goji (Lycium spp.) by the natural standard research collaboration. J Diet Suppl 12: 184-240.

3. Ruffo M, Parisi OI, Amone F, Malivindi R, Gorgoglione D, et al.(2017) Calabrian goji vs chinese goji: A comparative study on biological properties. Foods6: 1-10.
4. Xia G, Xin N, Liu W, Yao H, HouY, et al. (2014) Inhibitory effect of Lycium barbarum polysaccharides on cell apoptosis and senescence is potentially mediated by the p53 signaling pathway. Mol Med Rep 9: $1237-1241$.

5. Lau BWM, Lee JCD, LI Y, Fung SMY, Sang YH, et al. (2012) Polysaccharides from wolfberry prevents corticosterone-induced inhibition of sexual behavior and increases neurogenesis. PloS ONE 7: e33374.

6. Wang T, Li W, Wang Y, Zhou R, Ma L et al. (2014) Lycium barbarum polysaccharide prevents focal cerebral ischemic injury by inhibiting neuronal apoptosis in mice. Plos One 9: e-90780.

7. Gao K, Liu M, Cao J, Yao M, Lu Y, et al. (2015) Protective effects of Lycium barbarum polysaccharide on 6-OHDA-induced apoptosis in PC12 cells through the ROS-NO pathway. Molecules 20: 293-308.

8. Amagase H, Nance DM (2008) A randomized double-blind placebocontrolled clinical study of the general effects of a standardized Lycium barbarum (goji) juiceGoChi. J Altern Complement Med 14: 403-412.

9. Tang WM, Chan E, Kwok CY, Lee YK, Wu JH, et al.(2012) A review of the anticancer and immunomodulatory effects of Lycium barbarum fruit. Inflammopharmacology20: 307-314.

10. Ceccarini MR, Vannini S, Cataldi S, Moretti M, Villarini M, et al.(2016) In vitro protective effects of Lycium barbarum berries cultivated in Umbria (Italy) on human hepatocellular carcinoma cells. BioMed Res Int2016:7529521.

11. Zhang XJ, Yu HY, Cai YJ, Ke M (2017) Lycium barbarum polysaccharides inhibit proliferation and migration of bladder cancer cell lines BIU87 by suppressing Pi3K/AKT pathway. Oncotarget 8: 5936-5942.

12. Zhao R, Jin R, Chen $Y$, Han F (2015) Hypoglycemic and hypolipidemic effects of Lycium barbarum polysaccharide in diabetic rats. Chi Herb Med7: 310-315.

13. Kim MH, Lee JE, Lee JS, Yang WM (2016) Improvement of osteoporosis by Lycium chinese administration in ovariectomized mice. J Chin Med Assoc 80: 222-226.

14. Park SY, Park WT, Park YC, Ju JI, Park SU, et al. (2012) Metabolomics for the quality assessment of Lycium chinense fruits. BiosciBiotechnolBiochem76: 2188-2194.

15. Zhu J, Liu W, Yu J, Zou S, Wang J (2013) Characterization and hypoglycemic effect of a polysaccharide extracted from the fruit of Lycium barbarum. CarbohydrPolym 98: 8-16.

16. Amagase $H$, Sun B, Borek C (2009) Lycium barbarum (goji) juice improves in vivo antioxidant biomarkers in serum of healthy adults. Nutr Res 29: 19-25.

17. Brazilian Society of Diabetes. Guidelines 2015-2016.

18. Sposito AC, Caramelli B, Fonseca FA, Bertolami MC, AfluneNeto A, et al. (2007) IVBrazilian Guideline on dyslipidemia and prevention of atherosclerosis: Department of Atherosclerosis of the Brazilian Society of Cardiology. Arq Bras Cardiol 88: 2-19.

19. Amagase H, Nance DM (2011) Lycium barbarum increases caloric expenditure and decreases waist circumference in healthy overweight men and women Pilot Study. J Am Coll Nutr 30: 304-309.

20. De Koning L, Merchant AT, Pogue J, Anand SS (2007) Waist circumference and waist-to-hip ratio as predictors of cardiovascular events: meta-regression analysis of prospective studies. Eur Heart J 28: 850-856. 


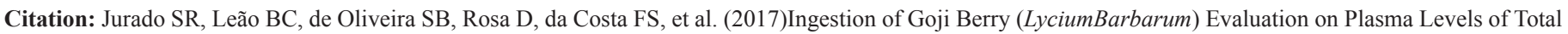
Cholesterol, Lipid Fractions, Glycaemia, Serotonin and Arterial Pressure. Cardiolog Res Cardiovasc Med 2: 116. DOI: 10.29011/2575-7083.000016

21. Luo Q, Cai Y, Yan J, Sun M, Corke H (2004) Hypoglycemic and hypolipidemic effects and antioxidant activity of fruit extracts from Lycium barbarum. Life Sci 76: 137-149.

22. Jing J, Cui G, Feng Q, Xiao Y (2009) Evaluation of hypoglycemic activity of the polysaccharides extracted from Lycium barbarum. Afr J Trad Complement Altern Med 6: 579-584.

23. Zou S, Zhang X, Yao W, Niu Y, Gao X (2010) Structure characterization and hypoglycemic activity of a polysaccharide isolated from the fruit of Lycium barbarum. CarbohydrPolym 80: 1161-1167.

24. Cai H, Liu F, Zuo P, Huang G, Song Z, et al. (2015) Practical application of antidiabetic efficacy of Lycium barbarum polysaccharide in patients with type 2 diabetes. Med Chem 11: 383-390.

25. Catalamo G, Guerin M (2010)The HDL Handbook Biological functions and clinical implications. Academic Press,USA.

26. Del Aguila LF, Claffey KP, Kirwan JP (1999) TNF-alpha impairs insulin signaling and insulin stimulation of glucose uptake in $\mathrm{C} 2 \mathrm{C} 12$ muscle cells. Am JPhysiol 276: E849-855.

27. Jing J, Cui G, Feng Q, Xiao Y (2009) Orthogonal test design for optimization of the extraction of polysaccharides from Lycium barbarum and evaluation of its anti-athletic fatigue activity. J Med Plants Res3: 433-437.
28. Kamakura M, Mitani N, Fukuda T, Fukushima, M (2001) Antifatigue effect of fresh Royal jelly in mice. J Nutr Sci Vitaminol47: 394-401.

29. Zhao Z, Luo Y, Li G, Zhu L, Wang Y, et al. (2013) Thoracic aorta vasoreactivity in rats under exhaustive exercise effects of Lycium barbarum polysaccharides supplementation. J IntSoc Sports Nutr 10: 1-7.

30. Moon WM, Park JW, Lee KW, Jeong HC, Choi J.B, et al. (2016) Administration of goji (Lycium chinense Mill.) extracts improves erectile function in old aged rat model. World J Mens Health 35: 43-50.

31. Kurkin VA, Dubishchev AV, Ezhkov VN, Titova IN, Avdeeva EV (2006) Antidepressant activity of some phytopharmaceuticals and phenylpropanoids. Pharm Chem J 40: 614-619.

32. Muszyńska B,Łojewski M,Rojowski J,Opoka W,Sułkowska-Ziaja K (2015)Natural products of relevance in the prevention and supportive treatment of depression. Psychiatr Pol49: 435-453.

33. Amagase H, Nance DM (2009) Effects of Lycium barbarum on feelings of general well-being, neurological/psychological traits and plasma levels of catecholamines A Randomized Double-blind Placebo-controlled Human Clinical Study. FASEB J23: 716.

34. Hsu CHP, Nance DM, Amagase H (2012) A meta-analysis of clinical improvements of general well-being by a standardized Lycium barbarum. J Med Food 15: 1006-1014. 